Revista Interdisciplinaria de Humanidades, Educación, Ciencia y Tecnología

Año VII. Vol. VII. N³3. Edición Especial III. 2021

Hecho el depósito de ley: pp201602FA4721

ISSN-L: 2542-3029; ISSN: 2610-802X

Universidad Nacional Experimental Francisco de Miranda (UNEFM). Santa Ana de Coro. Venezuela

Gabriela del Rocio Yumbla-Sanchez; Ana Zulema Castro-Salazar

DOI $10.35381 / \mathrm{cm} . \mathrm{v} 7 \mathrm{i} 3.576$

\title{
Google Classroom una herramienta alternativa de interaprendizaje en el área de ciencias sociales
}

\section{Google Classroom is an alternative tool for interlearing in social studies}

\author{
Gabriela del Rocio Yumbla-Sánchez \\ gabriela.yumbla.91@est.ucacue.edu.ec \\ Universidad Católica de Cuenca, Santa Isabel \\ Ecuador \\ https://orcid.org/0000-0002-7033-0967 \\ Ana Zulema Castro-Salazar \\ azcastros@ucacue.edu.ec \\ Universidad Católica de Cuenca, Azogues \\ Ecuador \\ https://orcid.org/0000-0002-3837-314X
}

Recibido: 15 de agosto de 2021

Aprobado: 15 de noviembre de 2021 


\title{
RESUMEN
}

Se realizó la investigación con el objetivo de analizar de qué manera se puede usar Google Classroom como entorno virtual de aprendizaje para la enseñanza del área de sociales en la Unidad Educativa "EL PAN". La muestra poblacional estuvo conformada por 34 estudiantes del primero de Ciencias Básicas a quienes se les aplico una encuesta, mediante instrumento tipo cuestionario de 8 ítems y fue validada en el coeficiente de fiabilidad de alfa de Cronbach con el 0,794. Ha sido evidente que el aprendizaje a través del empleo de una plataforma educativa virtual podría ofrecer algunas ventajas desde una mayor flexibilidad de horarios, también una diversidad de recursos superior y especialmente la posibilidad de poder colaborar o realizar un intercambio de experiencias para un mejor desarrollo de competencias digitales.

Descriptores: Aprendizaje activo; método de aprendizaje; aprendizaje semipresencial. (Palabras tomadas del Tesauro UNESCO).

\begin{abstract}
The research was carried out with the aim of analyzing how Google Classroom can be used as a virtual learning environment for teaching the social area in the "EL PAN" Educational Unit. The population sample was made up of 34 students from the first year of Basic Sciences to whom a survey was applied using an 8-item questionnaire-type instrument and was validated in the Cronbach's alpha reliability coefficient with 0.794 . It has been evident that learning through the use of a virtual educational platform could offer some advantages from a greater flexibility of schedules, also a greater diversity of resources and especially the possibility of being able to collaborate or carry out an exchange of experiences for a better development of digital skills.
\end{abstract}

Descriptors: Activity learning; learning methods; blended learning. (Words taken from the UNESCO Thesaurus).

\section{INTRODUCCIÓN}


La educación en estos tiempos ha sufrido un cambio radical debido a la pandemia covid19 a nivel mundial, es por ello que muchas de las instituciones educativas no solo en nuestro país sino alrededor del mundo optaron por implementar una variedad de estrategias que les permitan seguir manteniendo activo el proceso de enseñanza y no paralizar sus actividades, los docentes buscaron apoyo en herramientas tecnológicas que puedan contribuir en este proceso.

Con relación a esta problemática y de acuerdo a la Comisión Económica para América Latina y el Caribe CEPAL (2020), ha mencionado que la esfera de la educación, tras la emergencia sucedida que dio lugar al cierre global de cada una de las actividades educativas presencial, en las instituciones educativas de más de 190 países, con el fin de disminuir y prevenir la propagación de este virus como mitigar su impacto en la población menor de edad; de tal modo que se ha planteado que incluso previamente a la aparición de esta pandemia toda la situación social que atravesaba la región era deteriorada, debido al respectivo aumento del índice de pobreza y a su vez de pobreza extrema se ha persistido en las desigualdades y por ende un creciente descontento social. (pág. 21)

Asimismo en esta parte la UNESCO (2020) explica de manera explícita que al momento de no existir algún tipo de interacción física así como no tener contacto en un mismo entorno escolar de manera presencial cada docente tiene la obligación de desempeñar varios roles como apoyo en el proceso de enseñanza durante todo el tiempo que sea pertinente en la pandemia de manera que dichos alumnos tengan la capacidad de transformarse en creadores de su propio aprendizaje en diversos entornos, además que sean capaces de mantener un carácter líder de valores e integradores de recursos pero especialmente que mantengan una interacción social entre compañeros resaltando el objetivo fundamental de prepararlos a distancia tomando en cuenta la forma de compensar la ausencia de una relación física hasta ser los autores de sus recursos curriculares personales. 
Cabe mencionar que dicha herramienta, es aprovechada como un apoyo para mejorar la comunicación entre docentes y estudiantes, tanto dentro como fuera del aula; se estima que el proceso para poder incorporar la tecnología a los proceso educativos ayudara al desarrollo y dará oportunidad para que los estudiantes adquieran la mayor parte de conocimiento actual y que su interés por el mismo sea superior, posteriormente crece la posibilidad de aclarar dudas con mucha más facilidad.

Adicionalmente Peña \& Herrera, (2020) en referencia a la INEC el año anterior logro determinar tras la interpretación de sus datos estadísticos que fueron facilitados entre los dos años recientes en nuestro país, que existe un $11,5 \%$ de su variación anual, asimismo el uso de una red inalámbrica desde el 2019 ha aumentado con un 59,2\% hasta un 70,7\% actualmente; por lo que se demostró que el desconocimiento digital se pronunció con un $-1,2 \%$ del año último.

Es así que algunos autores sobre esta problemática concuerdan, que para poder lograr un aprendizaje no presencial de manera exitosa con los estudiantes es necesario totalmente el empleo de ciertos sistemas y diseños pedagógicos correctos por lo que es debido que los docentes sepan enfrentarse hacia una infinidad de desafíos en esta situación que no son comunes pero que, sin embargo, sean útiles para habilitar y ser implementados en su práctica de enseñanza.

De tal manera que se ha propuesto en esta investigación, centrarse en la aplicación de herramientas tecnológicas, como el uso de Google Classroom; como plataforma de apoyo dentro de los procesos de aprendizaje en el área de Ciencias Sociales, en la Unidad Educativa "El Pan", lo cual además servirá para poder rectificar el ausentismo escolar, mejorando así la comunicación entres docentes y estudiantes, ya que la mayor parte de familias en el cantón el Pan cuentan con redes de informáticas.

\section{Referencial teórico}

La educación en estos tiempos ha sufrido un cambio radical debido a la pandemia COVID- 19 a nivel mundial, es por ello que muchas de las instituciones educativas no 
solo en nuestro país sino alrededor del mundo optaron por implementar una variedad de estrategias que les permitan seguir manteniendo activo el proceso de enseñanza y no paralizar sus actividades, los docentes buscaron apoyo en herramientas tecnológicas que puedan contribuir en este proceso.

Con relación a esta problemática y de acuerdo a la Comisión Económica para América Latina y el Caribe NU. CEPAL (2020) ha mencionado que la esfera de la educación, tras la emergencia sucedida que dio lugar al cierre global de cada una de las actividades educativas presencial, en las instituciones educativas de más de 190 países, con el fin de disminuir y prevenir la propagación de este virus como mitigar su impacto en la población menor de edad; de tal modo que se ha planteado que incluso previamente a la aparición de esta pandemia toda la situación social que atravesaba la región era deteriorada, debido al respectivo aumento del índice de pobreza y a su vez de pobreza extrema se ha persistido en las desigualdades y por ende un creciente descontento social. (pág. 21)

Asimismo en esta parte la UNESCO (2020) explica de manera explícita que al momento de no existir algún tipo de interacción física así como no tener contacto en un mismo entorno escolar de manera presencial cada docente tiene la obligación de desempeñar varios roles como apoyo en el proceso de enseñanza durante todo el tiempo que sea pertinente en la pandemia de manera que dichos alumnos tengan la capacidad de transformarse en creadores de su propio aprendizaje en diversos entornos, además que sean capaces de mantener un carácter líder de valores e integradores de recursos pero especialmente que mantengan una interacción social entre compañeros resaltando el objetivo fundamental de prepararlos a distancia tomando en cuenta la forma de compensar la ausencia de una relación física hasta ser los autores de sus recursos curriculares personales.

Cabe mencionar que dicha herramienta, es aprovechada como un apoyo para mejorar la comunicación entre docentes y estudiantes, tanto dentro como fuera del aula; se estima que el proceso para poder incorporar la tecnología a los proceso educativos ayudara al 
desarrollo y dará oportunidad para que los estudiantes adquieran la mayor parte de conocimiento actual y que su interés por el mismo sea superior, posteriormente crece la posibilidad de aclarar dudas con mucha más facilidad.

Adicionalmente Peña \& Herrera (2020) logro determinar tras la interpretación de sus datos estadísticos mencionados de acuerdo a la INEC y que a su vez fueron facilitados entre los dos años recientes en nuestro país, que existe un 11,5\% de su variación anual, asimismo el uso de una red inalámbrica desde el 2019 ha aumentado con un 59,2\% hasta un $70,7 \%$ actualmente; por lo que se demostró que el desconocimiento digital se pronunció con un $-1,2 \%$ del año último.

Es así que algunos autores sobre esta problemática concuerdan, que para poder lograr un aprendizaje no presencial de manera exitosa con los estudiantes es necesario totalmente el empleo de ciertos sistemas y diseños pedagógicos correctos por lo que es debido que los docentes sepan enfrentarse hacia una infinidad de desafíos en esta situación que no son comunes pero que; sin embargo, sean útiles para habilitar y ser implementados en su práctica de enseñanza.

De tal manera que se ha propuesto en esta investigación centrarse en la aplicación de herramientas tecnológicas, como el uso de Google Classroom; como plataforma de apoyo dentro de los procesos de aprendizaje que se dan en la Unidad Educativa "EI Pan", lo cual además servirá para poder rectificar el ausentismo escolar, mejorando así la comunicación entres docentes y estudiantes, ya que la mayor parte de familias en el cantón el Pan cuentan con redes de informáticas.

En los últimos cinco años la implementación de tecnologías en el ámbito educativo ha logrado imponerse de manera considerable, tras la pandemia de covid-19 muchas instituciones, optaron por educación virtual, para realizar este proyecto para realizar la investigación bibliográfica se tomará una recopilación de artículos científicos obtenidos a través de bases de datos seguras como Scielo y Redalyc partiendo desde una perspectiva geográfica a nivel global y regional. 
Expresan Zamzami y Siti-Hajar (2016) en Malasia, explican tras una de sus investigaciones con respecto al análisis, tendencias y contenidos dentro del aula que el Aula Invertida que ha sido fundamental para la práctica de una buena enseñanza ya que el rol que cumple el docente debería ser constituido y armonizado con todas las condiciones de aprendizaje del alumno; en cuya investigación se deslindaron resultados esperados donde manifestaban que el educador deberá inmiscuir algunas estrategias que permitan la mejora de su educación y que a su vez se ajuste al estilo de aprendizaje que posee cada alumno para que el mismo tenga un aprendizaje activo y efectivo de acuerdo a su propio ritmo.

De acuerdo a las aportaciones realizadas por Aguilera-Ruiz et al., (2017) en España, se logró determinar que al momento de emplear el modelo virtual de formación en la actualidad y alrededor de todo el mundo este tipo de aulas son las más manejadas por parte de los docentes considerando que se ha caracterizado por ser una ayuda fundamental en su enseñanza impartida hasta dejar de lado lo que era la educación tradicional y modificándola por una educación más innovadora puesto que son los mismos estudiantes los que construyen sus conocimientos de manera más activa y pudiendo atender sus propias necesidades individuales.

Asimismo en una de las investigaciones realizadas por Kraus et al., (2019) en España, mencionan que los actores tomados en cuenta en su estudio se encontraban enlazados al uso estratégico de este tipo de plataforma digital dentro del contexto del programa integral para la igualdad educativa dado que lo recibían como un espacio básico virtual en sus capacitaciones complementarias no presenciales y que por ende si aportaban enérgicamente a cada proceso de enseñanza y aprendizaje de manera ubicua.

Para Martinell-Ramirez y Casillas (2017) en México, en sus investigaciones realizadas manifiesta que toda cultura digital debe ser fundamental, para el mejoramiento en la comunicación que podrían mantener docentes y estudiantes dado que los mismos manipulan con más frecuencia el ámbito digital como el correo electrónico o redes sociales, siendo el resultado del aislamiento brindado a las tecnologías antiguas que eran 
limitadas; los resultados mostraron que al momento de agregar la tecnología en sus adaptaciones curriculares dentro de su práctica educativa se ha conseguido mejores resultados en su rendimiento académico y aprendizaje activo.

De la misma forma Hernandez (2017) en Perú, concluyó que el uso de las TIC sería preciso para integrarse a la sociedad digital que vivimos actualmente, siendo así que los docentes para poder aplicar dichas herramientas en su práctica de clase debe modificar de acuerdo a su metodología empleada de forma diaria y que también requerirá la capacitación correcta para afrontar estos retos nuevos en la educación. Al aplicar esta iniciativa adecuadamente se tendrá como resultados la transformación en un recurso totalmente valioso en el aprendizaje hasta la formación de alumnos con capacidades personales profesionales idóneas para el desarrollo de un país.

Igualmente Pastora-Alejo y Fuentes-Aparicio (2020) en Ecuador, menciona que el progreso de la tecnología ha favorecido de forma representativa al ámbito educativo, gracias a que ha roto todos los paradigmas que se han mantenido de la educación tradicional, es por ello que hoy en día los docentes se acomodaran a cada una de las habilidades investigativas que sean sólidas en conocimientos de asignaturas específicas obteniendo una comunicación eficaz entre docentes y estudiantes para el respectivo manejo de su grupo.

De acuerdo a Gomez-Goitia (2020) en Ecuador, se puedo determinar, que la educación a nivel nacional al fin consiguió un logro importante, al aplicar tecnologías educativas en los procesos de enseñanza-aprendizaje puesto que mediante esta herramienta los docentes podrán compartir libros y materiales digitales que anteriormente no se encontraban disponibles; de acuerdo a esta aplicación de Classroom cada uno de los centros de estudio tendrán la capacidad de innovar aquellas herramientas que se emplean en la gestión didáctica por lo que se ha logrado un interés superior por participar y elaborar tareas de manera dinámica.

Finalmente, Argandoña-Mendoza et al. (2020) en Ecuador, realizo acciones investigativas en las que sustenta que se debe acomodar a los nuevos paradigmas de la 
etapa tecnológica, la misma que se basó en el análisis de varias teorías pedagógicas las mismas que se han determinado según varias formas de enseñar y aprender como es el caso de la Classroom conocida por potencializar la creación de temas en la clase de forma virtual, asimismo cabe destacar que con el uso adecuado de estas tecnologías por parte de los alumnos se obtendrá un buen rendimiento académico permitiendo a los estudiantes el poder trabajar en la plataforma en diferentes horarios.

Es así que dichos autores planteaban una nueva incorporación de medios tecnológicos en el ámbito de la educación para obtener como resultado principal favorecería a lo que sería el aprendizaje ubicuo o mejor conocido como una educación llevada a cabo en cualquier lugar o momento; siendo así pertinente el replanteamiento de un escenario netamente nuevo hasta poder pensar algunas propuestas en la enseñanza, aplicando en sus clases el uso de las herramientas tecnológicas para incentivar y motivar a los estudiantes.

De acuerdo a Arias y Restagno (2014) se ha conocido que Google Classroom ha sido considerada como una plataforma netamente virtual educativa que no exige ningún tipo de aporte económico, que fue originado por blendeg learning como empresa de Google es por ello que podría brindar la oportunidad de continuar con un aprendizaje aunque este sea de forma semipresencial; dicha aplicación tiene como funciones relevantes la facilidad de simplificar y distribuir trabajos con agilidad hasta que el docente pueda evaluar este contenido al instante. Además, permite dar apertura a nuevas aulas virtuales dentro de una Institución educativa, así como el poder facilitar el trabajo a los miembros de la comunidad académica fuera de la institución.

Por otra parte esta plataforma de Google que ha sido modificada en beneficio de la educación ha sido tomada como una de las más relevantes en la actualidad debido a que con esta herramienta que mantiene un uso totalmente versátil en el campo académico se podrá exponer una variedad de ventajas dentro del entorno virtual educativo; entre sus ventajas está el ahorro permanente de un tiempo considerable así como de utilería que sería empleada en las clases presenciales, es así que en el caso de los educadores 
tienen acceso a la jornada educativa diaria sin tener contacto físico y por ende pueden receptar las tareas y evaluación manteniendo una comunicación organizada a cualquier hora prevista.

Como complemento se ha tomado a Chiriguaya-Morales et al., (2018) el mismo que ha mencionado que un entorno virtual sería un espacio educativo que ha esta albergado en una página u aplicación web, ostenta diversas herramientas de tipo informático brinda la posibilidad de compartir didácticamente entre estudiantes y docentes; cada uno de ellos tienen la oportunidad de llevar a cabo acciones sean estas propias de la docencia o de la educación entre los cuales estarían un conversatorio, lectura de documentos, evaluaciones, trabajo colaborativo e individuales cada una de estas de modo virtual.

Otro de los aspectos importantes en la investigación seria la práctica de la didáctica con un toque de tecnología, por lo que sería conveniente precisar cada uno de los aportes y sus repercusiones de todo este avance por lo que requerirá cierto conocimiento previo siempre y cuando se centre en el mundo educativo; esta conceptualización ha sido definida directamente en la didáctica y su forma de manejarse en la respectiva relación con la tecnología educativa. En este punto, una variedad de autores han logrado desarrollar dicha posición la misma que no podría ir más allá de conocimiento básico sino que también ayude a replantearse las dudas con respecto a las funciones que deberán cumplir los educadores en conjunto con toda la comunidad educativo.

Tras este aspecto se ha establecido un análisis total de los medios y el tipo de avance alcanzado al momento de ser un objeto de estudio y de haber sido suplido correctamente en la enseñanza general; para lo cual se ha tomado en cuenta cuáles son sus contenidos referidos con la cultura digital en su jornada diaria de clase. Para la enseñanza adecuada se ubicaron considerablemente las herramientas propuestas al manejo de la generación en situaciones de enseñanza-aprendizaje y a su vez el respectivo desarrollo personal de cada uno de los miembros de la comunidad educativa participe; posteriormente se hará énfasis en la elección y uso adecuado de la plataforma empleada. 
En concordancia con lo antes mencionado se refiere a una plataforma digital gratuita que da la apertura al uso de manera ilimitada de los servicios que este facilita, logrando acceder fácilmente gracias a una rápida conexión en los servicios integrados de google; de tal forma que cada una de sus funciones sean un complemento dirigido a la educación con aspectos prominentes, por lo que es posible la realización de trabajos colaborativos para compartirlos continuamente con cada estudiante dentro de un grupo colaborativo y con supervisión del docente.

Esta nueva realidad que el mundo educativo atraviesa conlleva cierta complejidad para que desarrollen las habilidades los alumnos, por lo que es previsor buscar recursos académicos e informativos para ayudar a mejorar su rendimiento con la aplicación de actividades específicas en entornos digitales. De manera que con esta aplicación se cree un vínculo empático de parte del educador con el alumno siendo importante en muchos de los casos forjarlo hasta convertirse en un campo humano netamente fraterno; aunque a su vez este se convertirá en uno de los aspectos negativos al momento de cruzar la línea de lo profesional a un vínculo más afectivo tomando en cuenta que en la actualidad no existe una convivencia diaria dentro de un espacio físico, sino que se basa específicamente en el uso común de una herramienta digital.

Según la UNESCO (2013) en uno de sus artículos publicados referentes a los enfoques estratégicos sobre los medios digitales dentro de la educación en América Latina y el Caribe han manifestado que la incorporación de aparatos tecnológicos o plataformas digitales en las aulas de clase podría suponer una imponencia en la necesidad global de una nueva definición de los roles a cumplir por parte de los docentes y estudiantes; de tal forma que los primeros favorecidos en la adquisición de estas nuevas herramientas sean cada uno de los integrantes de la comunidad educativa, gracias a que adquieren una autonomía y responsabilidad superior en el proceso de aprendizaje hasta tener como resultado por un lado la separación del rol del docente clásico como fuente única de un conocimiento. 


\section{METODOLOGÍA}

Para este proyecto se planteó trabajar mediante un enfoque mixto, desde un alcance descriptivo con un diseño no experimental transversal (Erazo, 2021). Según Salas y Mata (2020) será fundamental motivar el uso compartido de los enfoques cualitativos y cuantitativos como parte útil que aprovecha cada fortaleza que brinda un enfoque de investigación obteniendo así estudios más profundos que abarquen las realidades educativas.

De acuerdo con Hernandez-Ramirez (2012) todas las investigación de origen científico deben contener un puente entre lo que es el problema y la solución ya que nos ayudara a mejorar las respuestas de las hipótesis plateadas objetivamente, por ello se utilizó un diseño no experimental.

Según lo mencionado por Orellana-Lopez y Sanchez-Gomez (2006) en relaciòn a los entornos virtuales generados con gran potencial y que de manera convensional modifican los procedimientos o tecnica para la recoleccion de datos en aspectos como es el caso de analisis es por ello que se ha propuesto como tecnica de invetigacion la encuesta a estudiantes y entrevista a docentes:

En concordancia con Lopera-Echavarria et al., (2010), se aplicó el metodo analitico gracias a que da cuenta del objeto de estudio dentro de una problemática de investigación, en la investigación se realizara un análisis de toda la información recogida en los datos primarios y secundarios para a su vez procesarlos e interpretarlos. En la parte de análisis de datos de tendrá en consideración:

- Recolección de los datos tras la aplicación de las técnicas e instrumentos respectivos.

- Expectación de la información obtenida.

- Procesamiento de datos con la utilización de aplicaciones informáticas.

- Tabulación y posteriormente representación gráfica de la información.

- Análisis e interpretación de los datos obtenidos. 
Con la finalidad de cumplir con el objetivo de Analizar de qué manera se puede usar Google Classroom como entorno virtual de aprendizaje para la enseñanza del área de sociales en la Unidad Educativa "EL PAN". La muestra poblacional estuvo conformada por 34 estudiantes del primero de Ciencias Básicas a quienes se les aplico una encuesta, mediante instrumento tipo cuestionario de 8 ítems y fue validada en el coeficiente de fiabilidad de alfa de Cronbach con el 0,794.

Un segundo segmento poblacional estuvo conformado por 9 docentes que conforman el área de Ciencias Sociales a quienes se les aplico una entrevista, mediante un instrumento tipo cuestionario de 7 ítems. Ambas muestras pertenecen a la Unidad Educativa "EL PAN" en el cantón el Pan-Ecuador, mediante la aplicación de encuentas a estudiantes y entrevistas a docentes, busco encontrar como es la utilización del Google classroom es el área de ciencias sociales.

Los datos fueron recolectados a través de Google forms, que fueron enviados por correo electrónico y WhatsApp, durante el periodo lectivo 2021-2022 en el mes de octubre. Analizándose la información recopilada, por medio de la estadística descriptiva, y chi cuadrado con apoyo del programa estadístico SPSS .

\section{RESULTADOS}

\section{Resultados Cuantitativos}

Cada uno de los resultados que se han logrado obtener, tras el procesamiento de datos a nivel cuantitativo, en relación a la encuesta aplicada, cabe destacar que se han plasmado mediante un proceso estadístico de forma descriptiva con el uso de SPSS; a su vez se tomara en cuenta cada uno de los parámetros establecidos previamente conjuntamente con los valores considerados aceptables en la prueba de Shapiro- Wilk dando como resultado que todas las variables son Paramétricas, de la misma forma para el análisis de los datos cualitativos se ha tomado como referencia Chi cuadrado al momento de realizar las siguientes hipótesis: 
HO: La utilización de Google Classroom como plataforma para el entorno virtual de aprendizaje ha traído resultados positivos en la enseñanza del área de Ciencias Sociales en los estudiantes.

H1: La utilización de Google Classroom como plataforma para el entorno virtual de aprendizaje No ha producido resultados positivos en la enseñanza del área de Ciencias Sociales en los estudiantes.

\section{Tabla 1.}

Uso de plataformas en las asignaturas del area de Ciencias Sociales .

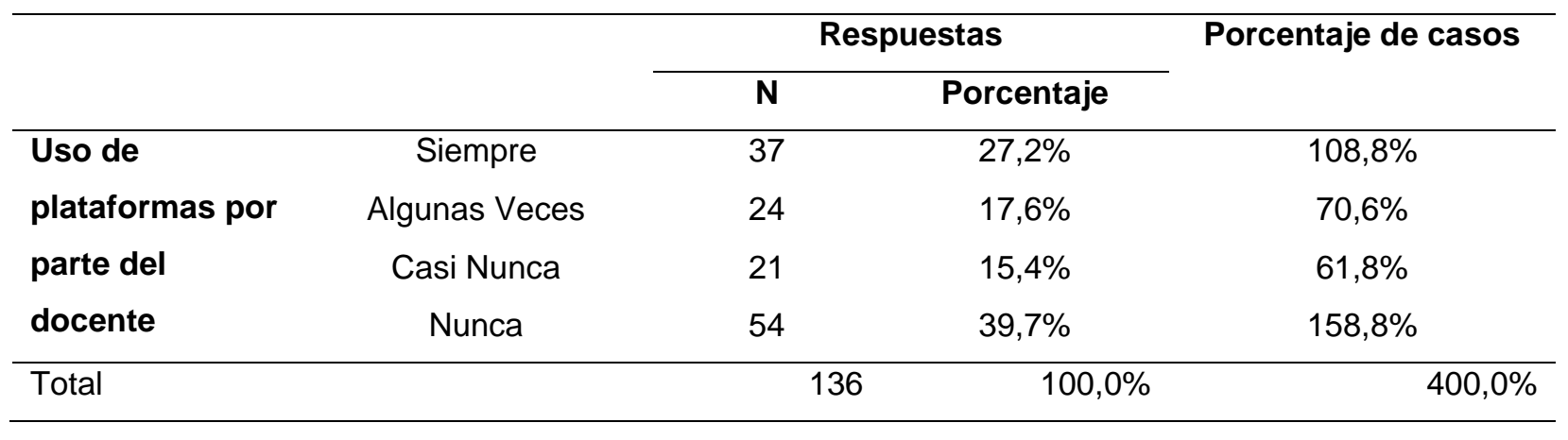

Fuente: Encuesta.

Una vez realizado el respectivo análisis de la relación que existe entre las variables tomadas en cuenta en el estudio como la Frecuencia que utilizan los estudiantes, la utilización de esta plataforma hasta la eficiencia que podría conllevar el empleo de esta 
plataforma virtual en la enseñanza; no se logró establecer una diferencia superior gracias a que los valores que se obtuvieron tanto en Spss como el porcentaje ordinal para el Chi cuadrado superan el $10,01 \%$ por lo que se alega que nuestra hipótesis planteada es nula, haciendo énfasis que no existe ninguna relación entre las mismas.

\section{Tabla 2.}

Uso de plataformas Edmodo y Classroom.

\begin{tabular}{llrrr}
\hline & & \multicolumn{2}{c}{ Respuestas } & \multicolumn{2}{c}{$\begin{array}{c}\text { Porcentaje de } \\
\text { casos }\end{array}$} \\
\cline { 3 - 4 } & & $\mathrm{N}$ & Porcentaje & $100,0 \%$ \\
\hline Uso de & Siempre & 34 & $25,0 \%$ & $85,3 \%$ \\
plataformas & Algunas veces & 29 & $21,3 \%$ & $55,9 \%$ \\
Edmodo y & Casi nunca & 19 & $14,0 \%$ & $158,8 \%$ \\
Classroom & Nunca & 54 & $39,7 \%$ & $400,0 \%$ \\
\hline Total & & 136 & $100,0 \%$ & \\
\hline
\end{tabular}

Fuente: Encuesta

En esta sección es evidente el ratificar que un 39,7\% de los estudiantes encuestados han manifestado que la plataforma digital que nunca han utilizado seria Edmodo como parte en el proceso de enseñanza o retroalimentación de cada clase impartida; además se ha manifestado que la única aplicación que se utiliza en el área de Ciencias Sociales es Classroom el mismo que ha sido representado con un $25 \%$.

\section{Tabla 3.}

Uso de plataforma Classroom.

\begin{tabular}{llrrr}
\hline & & \multicolumn{2}{c}{ Respuestas } & \multicolumn{2}{c}{$\begin{array}{c}\text { Porcentaje de } \\
\text { cann }\end{array}$} \\
\cline { 3 - 4 } & & $\mathrm{N}$ & Porcentaje & \multicolumn{1}{c}{ casos } \\
\hline Uso de & Siempre & 131 & $77,1 \%$ & $108,3 \%$ \\
plataforma & Algunas veces & 37 & $21,8 \%$ & $2,9 \%$ \\
Classroom & Casi nunca & 1 & $0,6 \%$ & $2,9 \%$ \\
& Nunca & 1 & $0,6 \%$ & $500,0 \%$ \\
\hline Total & & 170 & $100,0 \%$ & \\
\hline
\end{tabular}




\section{Fuente: Encuesta}

Asimismo se ha podido observar en la siguiente interrogante que un $77 \%$ de los estudiantes encuestados han demostrado que Siempre utilizan alguna plataforma digital en este caso Classroom sería más utilizado al momento de realizar sus tareas en la actualidad, por otro lado se ha manifestado la opinión de los alumnos con un $0,6 \%$ que han considerado que Nunca se debe dejar de utilizar este tipo de plataformas, de manera contraria creen que sería fundamental el empleo de ciertas aplicaciones en su entorno educativo.

\section{Resultados Cualitativos}

Una vez tomado en consideración en esta investigación el grupo de 9 docentes que ejercen su labor diaria en el área de Ciencias Sociales, se procedió a crear una reunión de manera virtual con cada uno de los mismos, así también se propuso el mantener constante comunicación para desarrollar la entrevista individual propuesta en el siguiente estudio; no obstante, se tomó en consideración las condiciones y medidas actuales por lo que se dio paso a la misma de manera online con una duración de más o menos 15 a 30 minutos para cada docente, brindando la facilidad de exponer su respuesta de manera espontánea.

\section{Tabla 4.}

Interpretación de resultados cualitativos.

\begin{tabular}{|c|c|c|}
\hline $\begin{array}{l}\text { Unidad de } \\
\text { Análisis }\end{array}$ & Categoría & Segmento \\
\hline $\begin{array}{l}\text { Plataformas } \\
\text { Educativas }\end{array}$ & & $\begin{array}{l}\text { Ha sido evidente en el conocimiento que portan los } \\
\text { docentes acerca de esta plataforma digital, aunque a su }\end{array}$ \\
\hline
\end{tabular}


Conocimientos sobre plataformas educativas

Google Classroom

Utilización de Google Classroom

Herramientas Tecnológicas

Incorporación

\section{Incorporación de Google Classroom}

vez se debe recalcar el auto-aprendizaje tomando en cuenta que previo a esta pandemia mundial no se manejaban dichas plataformas en su labor educativa diaria; sin embargo han sido fundamentales ya que se han tomado como herramientas de transferencia de un mismo conocimiento y especialmente forjan una mejor adquisición, asimilación y construcción del conocimiento.

De igual manera se expone la necesidad de implementar este tipo de plataformas dentro de toda institución educativa, gracias a la facilidad que brindan tanto a los estudiantes como a los docentes a verificar su retroalimentación, además de incentivar el trabajo colaborativo según se adapte a su tiempo; tomando en cuenta siempre y cuando la motivación y guía por parte del docente hasta generar un autoconocimiento en los alumnos.

De manera general se considera como aporte fundamental la implementación de charlas o capacitaciones tanto para estudiantes como docentes acerca del correcto uso no solo de dicha plataforma sino de otras más que podrían beneficiar a futuro el aprendizaje y la enseñanza por parte de los educadores; también se considera la inserción de estrategias educativas como por ejemplo la gamificación cuya única finalidad seria conseguir un aprendizaje significativo en los alumnos.

En el ámbito educativo en su mayoría se ha considerado primordial la inserción de esta herramienta digital tanto al aprendizaje como a la enseñanza de los contenidos educativos, puesto que trata de una plataforma fácil y útil en uso; lo que generaría un beneficio al momento de entregar e integrarse a cada una de las actividades académicas hasta conocer las falencias existentes en las mismas, al mismo tiempo de beneficiar a los docentes a controlar y manejar un registro eficaz de los valores académicos a nivel global. Inicialmente se debe tomar en cuenta que un aprendizaje significativo trata de una construcción constante entre lo brindado por el docente y el alumno, es así que sería adecuado el uso constante de esta aplicación no en su mayoría para dicho aprendizaje sino más bien como un refuerzo o apoyo hacia esta 
construcción, insistiendo una vez más en la capacidad de manejarla, siendo así necesario su uso no solamente en el área de Sociales sino en muchas áreas educativas más; asimismo teniendo en consideración la atracción que causa y la mejora en la comprensión del conocimiento.

En la actualidad, el manejo de esta plataforma conlleva la capacidad de compartir e interactuar con los estudiantes a través de documentos, links, videos, imágenes entre otras; siendo así fundamental el reforzar cada actividad diaria sea esta en la jornada de

Distribución documentos con esta plataforma clase como fuera de ella con varios documentos que apoyen su aprendizaje, por otra parte también se ha incentivado el trabajo colaborativo logrando el intercambio de opiniones o pensamientos entre los mismos y por ende convirtiéndose en actores principales en la construcción de su propio conocimiento.

Se ha considerado a esta herramienta digital como una de las principales en la actualidad que debería o está siendo actor fundamental en la educación a nivel global de los estudiantes en cada nivel de educación, también se la ha tomado como apoyo siempre y cuando se Opinión Opinión sobre Google Classroom corresponsabilice el constante progreso del conocimiento diario; por lo mismo es la plataforma más practica al momento de interactuar y compartir documentos o a su vez al dar una calificación a las actividades escolares realizadas.

\section{Fuente: Entrevistas.}

En este aspecto se ha pronunciado la evidente influencia que posee en la actualidad Google Classroom en el ámbito educativo y especialmente en el área de Ciencias Sociales $u$ otras asignaturas, gracias a que se basa a encaminar hacia un aprendizaje significativo en los alumnos, además de la oportunidad de adquirir mayores capacidades en trabajo colaborativo y por ende reforzar sus habilidades para desenvolverse a futuro en la parte tecnológica. En relación a la presente investigación han existido algunos estudios similares que refuerzan los resultados obtenidos como por ejemplo la 
investigación realizada por Prado-Prado et al., (2020) donde se menciona que ante la pandemia acaecida en todo el mundo únicamente un 34\% de los estudiantes conocían el funcionamiento y manejan sus actividades escolares mediante una plataforma digital; ante esto sería prudente recomendar la implementación de acciones de refuerzo los mismos que aporten a su total aprendizaje.

Por otra parte, tenemos un estudio realizado por Cedeño-Escobar et al., (2020) en donde se pone de manifiesto que a consecuencia de la situación mundial por el covid-19 el ámbito de la educación ha sufrido grandes cambios implementando la tecnología, a tal punto que en nuestro país desde el Ministerio de Educación se incentivó la incorporación de varias plataformas digitales como Teams, Classroom, Drive, teniendo como resultado el poco conocimiento inicial de los estudiantes y docentes pese a que se trata de herramientas fáciles de manejar, procurando el autoaprendizaje de la comunidad educativa antes dichas plataformas digitales.

\section{PROPUESTA}

Ante la problemática identificada con esta investigación se desarrolló y se planteó como propuesta principal el uso de Google Classroom como herramienta digital para la enseñanza en el área de Ciencias Sociales en la Unidad Educativa "EL PAN"; con la única finalidad de brindar al estudiante una mayor facilidad es su aprendizaje, así como promover la enseñanza actual dinámica y significativa para el correcto aprendizaje de dicha asignatura. Como podemos observar en la figura 1, a partir desde un Plan estratégico que debería emplearse para nuestra propuesta se destaca cinco aspectos que se debe considerar: 


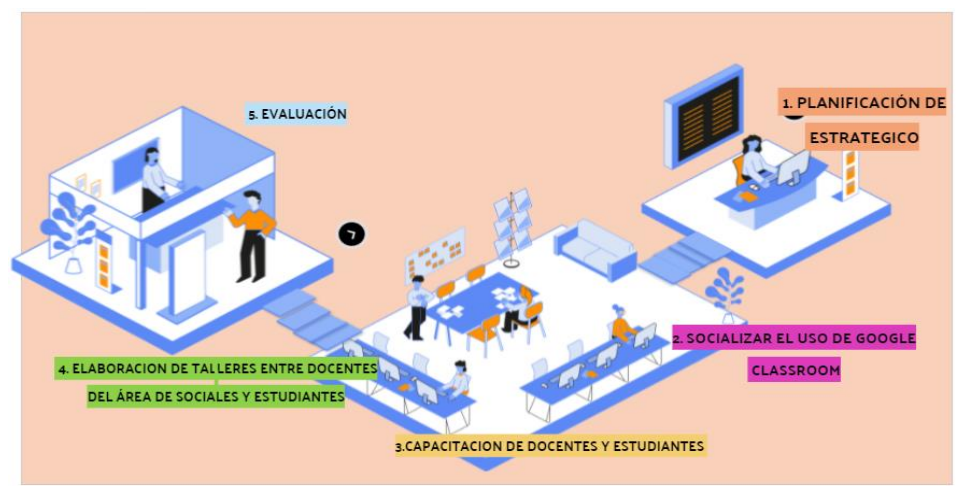

Figura 1. Propuesta de Plan Estratégico Elaboración: Los autores.

Los detalles de este plan estratégico se detallan a continuación:

- Implementación de la plataforma de Google Classroom como herramienta de apoyo para el proceso de enseñanza aprendizaje en el área de Ciencias Sociales

- Socializar el uso del Google Classroom como herramienta de apoyo.

- Capacitación al personal docente y estudiantes sobre la plataforma.

- Facilitar el acceso a la información requerida por el estudiante y además la facilidad de realizar tareas y trabajos de manera exitosa ante la educación en línea que atravesamos.

- Aplicar en el proceso de evaluación

No obstante, es preciso mencionar que esta propuesta atiende únicamente como sugerencia útil, tomando en cuenta que cada uno de los docentes se encuentra en la capacidad de desarrollar e implementar cualquier herramienta metodológica en su plan 
de estudio, siempre y cuando esta vele por el bienestar de todos los integrantes de la institución educativa.

Por consiguiente, se ha considerado pertinente plantear dicha propuesta con los alumnos y docentes que pertenecen al primero de bachillerato de la Unidad Educativa EL PAN de nuestra provincia, una vez que se ha destacado la gran importancia que se tiene en la actualidad el conocer el funcionamiento y utilización de estas plataformas digitales por parte no solo de los estudiantes sino de toda la comunidad educativa en general.

Ahora bien, en cuanto a los instrumentos empleados en nuestra propuesta que han sido fundamentales para el conocimiento de los resultados considerados por el grupo focal tenemos una encuesta principal compuesta de 3 preguntas principales, fragmentadas en respuestas múltiples; en el caso de los resultados cualitativos tenemos el empleo de una entrevista capaz de brindarnos el punto de vista de cada uno de los docentes entorno a nuestra investigación.

\section{CONCLUSIONES}

Al finalizar el siguiente trabajo investigativo se ha podido concluir fundamentalmente que esta plataforma educativa Google Classroom cumple una función relevante en el entorno del aprendizaje significativo virtual ya que ofrece un paquete de aplicaciones diseñadas para evolucionar la manera en que los educadores y los alumnos mantengas habilidades innovadoras donde aprendan y logren trabajar correctamente con las diversas herramientas gratuitas y seguras existentes; además de ello con dicho estudio se evidenció que los resultados obtenidos en el análisis de SPSS podrían ser adjudicados una gran parte de la responsabilidad a la falta de previsión y capacitación de los docentes por parte de la institución para implementar la plataforma y utilizar de manera positiva todas las herramientas que ofrece.

Adicionalmente tras la investigación, ha sido evidente que el aprendizaje a través del empleo de una plataforma educativa virtual podría ofrecer algunas ventajas desde una mayor flexibilidad de horarios, también una diversidad de recursos superior y 
especialmente la posibilidad de poder colaborar o realizar un intercambio de experiencias para un mejor desarrollo de competencias digitales.

Finalmente cabe mencionar que cada una de las plataformas digitales que existen brindan mayor facilidad en el ambiente de aprendizaje dando una adecuación hacia nuevas estrategias que permitirán un correcto avance cognitivo tanto con creatividad y diversión en lo que ya se consideraban áreas tradicionales en la malla curricular.

\section{REFERENCIAS CONSULTADAS}

Aguilera-Ruiz, C., Manzano-Leon, A., Martinez-Moreno, I., \& Lozano-Segura, M. (2017). El modelo flipped classroom. [The flipped classroom model]. INFAD Revista de Psicologia, 4(Informe), 261-266. https://n9.cl/210g

Arias, N., \& Restagno, A. (2014). Cultura digital, apropiación y educación: aportes para una didáctica transmedia. Estudio de caso. [Digital culture, appropriation and education: contributions for a transmedia didactics. Case study]. Revista Argentina de Comunicacion, 8(Informe), 110-141. https://n9.cl/ldchy

Argandoña-Mendoza, M., García-Mejía, R., Ayón-Parrales, E., \& Zambrano-Zambrano, Y. (2020). Investigación e innovación educativa: Reto escolar por COVID-19 en el $\begin{array}{lll}\text { Ecuador. EPISTEME KOINONIA, } & \text { 3(5), }\end{array}$ http://dx.doi.org/10.35381/e.k.v3i5.726

CEPAL-UNESCO. (2020). La Educación en tiempos de Pandemia de COVID- 19. [Education in times of the COVID-19 Pandemic]. In Comisión Económica para América Latina. https://n9.cl/b613e

Chiriguaya-Morales, M., Espinoza-Espinoza, M., \& Zamora, M. (2018). Google Classroom: vía para desarrollo del proceso de enseñanza-aprendizaje. [Google Classroom: way to develop the teaching-learning process] [Instituto Superior Tecnologico Bolivariano]. https://n9.cl/1wg9e

Erazo Álvarez, J. C. (2021). Capital intelectual y gestión de innovación: Pequeñas y medianas empresas de cuero y calzado en Tungurahua-Ecuador. Revista De Ciencias Sociales, 27, 230-245. Recuperado a partir de 
https://produccioncientificaluz.org/index.php/rcs/article/view/37004

Gomez-Goitia, J. (2020). Buena práctica docente para el diseño de aula virtual. [Good teaching practice for virtual classroom design]. Reviista Andina de Educacion, 3(Articulo), 64-66. https://doi.org/https://doi.org/10.32719/26312816.2020.3.1.7

Hernandez-Ramirez, R. (2012). Diseño de investigación transversal. [Cross-sectional research design]. https://n9.cl/y0l54

Hernandez, R. (2017). Impacto de las TIC en la educación. Retos y Perspectivas. [Impact of ICT on education. Challenges and Perspectives]. Impacto de Las TIC En La Educación, 5(Articulo de investigacion), 325-347. https://doi.org/http://dx.doi.org/10.20511/pyr2017.v5n1.149

Kraus, G., Formichela, M., \& Alderete, M. (2019). El uso de Google Classroom como complemento de la capacitación presencial a docentes de nivel primario. [The use of Google Classroom as a complement to face-to-face training for primary-level teachers]. Revista Iberoamericana de Tecnología En Educación, 24(Articulo), 10-13. https://doi.org/https://doi.org/10.24215/18509959.24.e09

Lopera-Echavarria, J., Ramirez-Gomez, C., Zuruaga-Arsitisabal, M., \& Ortiz-Vanegas, J. (2010). El método analítico como metodo natural. [The analytical method as a natural method]. Nómadas. Critical Journal of Social and Juridical Sciences, 25(Articulo), 210. https://n9.cl/ttp4x

Martinell-Ramirez, A., \& Casillas, M. (2017). Saberes digitales de los docentes en educación básica. [Digital knowledge of teachers in basic education]. Secretaría de Educación de Veracruz, 1(Informe), 108-127. https://n9.cl/wsdlr

NU. CEPAL. (2020). La educación en tiempos de la pandemia de COVID-19. [Education in times of the COVID-19 pandemic]. Comision Economica Para America Latina y EI Caribe. https://www.cepal.org/es/publicaciones/45904-la-educacion-tiempos-lapandemia-covid-19

Orellana-Lopez, D., \& Sanchez-Gomez, M. (2006). Técnicas de Recolección de Datos en Entornos Virtuales más usadas. [Data Collection Techniques in Virtual Environments 
most used]. Revista de Investigacion Educativa, 24(Informe), 205-222. https://n9.cl/6ageq

Pastora-Alejo, B., \& Fuentes-Aparicio, A. (2020). La planificación de estrategias de enseñanza en un entorno virtual de aprendizaje. [Planning teaching strategies in a virtual learning environment]. UISRAEL Revista Cientifica, 8(Informe), 59-76. https://doi.org/https://doi.org/10.35290/rcui.v8n1.2021.341

Peña, A., \& Herrera, L. (2020). Indicadores de tecnología de la información y comunicación. [Information and communication technology indicators]. INEC, 4(Boletin Tecnico), 13-15. https://n9.cl/gih4l

Prado-Prado, S., Garcia-Herrera, D., Erazo-Alvarez, J., \& Narvaez-Surita, C. (2020). Google Classroom: aplicacion educativa como entorno de aprendizaje en zonas rurales en contextos de Covid-19. [Google Classroom: educational application as a learning environment in rural areas in Covid-19 contexts]. Revista Arbitrada Interdisciplinaria KOINONIA, 5(Informe), 4-26. https://doi.org/http://dx.doi.org/10.35381/r.k.v5i5.1031

Salas, D., \& Mata, L. (2020). El Informe de Investigacion. Investigalia [The Investigation Report]. El Informe de Investigacion. https://n9.cl/ylnc0

UNESCO. (2013). Enfoques estratégicos sobre las TIC en educación de América Latina y el Caribe. [Strategic approaches to ICT in education in Latin America and the Caribbean]. Oficina Regional de Educaciónpara América Latina y El Caribe, 3(Informe), 14-47. https://doi.org/http://creativecommons.org/licenses/by-sa/3.0/igo/

UNESCO. (2020). Comision Economica para America Latina y el Caribe. La Educación En Tiempos de La Pandemia de COVID-19. https://n9.cl/oc77

Zamzami, Z., \& Siti-Hajar, H. (2016). Flipped Classroom research and trends from different fields of study. [Investigaciones y tendencias de Flipped Classroom de diferentes campos de estudio]. The International Review of Research in Open and Distributed Learning, https://doi.org/https://doi.org/10.19173/irrodl.v17i3.2274 
Revista Interdisciplinaria de Humanidades, Educación, Ciencia y Tecnología

Año VII. Vol. VII. N³. Edición Especial III. 2021

Hecho el depósito de ley: pp201602FA4721

ISSN-L: 2542-3029; ISSN: 2610-802X

Universidad Nacional Experimental Francisco de Miranda (UNEFM). Santa Ana de Coro. Venezuela

Gabriela del Rocio Yumbla-Sanchez; Ana Zulema Castro-Salazar

(C2021 por los autores. Este artículo es de acceso abierto y distribuido según los términos y condiciones de la licencia Creative Commons Atribución-NoComercial-Compartirlgual 4.0 Internacional (CC BY-NC-SA 4.0) (https://creativecommons.org/licenses/by-nc-sa/4.0/). 